Int. J. Dev. Biol. 56: 333-339 (2012)

doi: $10.1387 / \mathrm{ijdb} .113444 \mathrm{gx}$

\title{
Identification and characterization of ADAM41, a novel ADAM metalloproteinase in Xenopus
}

\author{
GUOFENG XU1', SHUO WEI*,1,2, JUDITH M. WHITE ${ }^{1}$ and DOUGLAS W. DESIMONE*,1 \\ ${ }^{1}$ Department of Cell Biology and the Morphogenesis and Regenerative Medicine Institute, University of Virginia, \\ Charlottesville, VA and ${ }^{2}$ Department of Biology, West Virginia University, Morgantown, WV, USA
}

\begin{abstract}
The ADAM family of transmembrane metalloproteinases has important functions in fertilization, development and disease, and is widely distributed throughout the Metazoa. In this study, we identified a novel ADAM protein in Xenopus tropicalis ( $X$. tropicalis) with closest overall sequence similarity to the Xenopus ADAM10 protein. Based on comparisons of available sequence information, putative orthologs of this ADAM (which we designate ADAM41) are identified in several other vertebrate species including non-placental mammals, but absent from placental mammals and aves. ADAM41 mRNA is maternally deposited in $X$. tropicalis with subsequent zygotic expression detected in the neural plate at neurula stages. Antisense morpholino knockdown of ADAM41 results in a delay in early neuronal marker expression, which can be rescued by a non-targeted ADAM41 transcript. Thus, ADAM41 is likely required for maintaining proper timing of neurogenesis in X. tropicalis.
\end{abstract}

KEY WORDS: ADAM, metalloproteinase, Xenopus, neurogenesis

\section{Introduction}

The ADAMs comprise a large family of membrane anchored proteins, characterized originally by the presence of a disintegrin and metalloproteinase domain (ADAM). Most ADAMs contain a prodomain and metalloproteinase, disintegrin and cysteine-rich domains facing the extracellular space, followed by a transmembrane domain and a cytoplasmic tail (Edwards et al., 2008; White et al., 2005). Accumulating evidence has revealed that ADAMs are associated with various cell signaling pathways through their multiple functional domains and that they mediate various pathological and normal physiological processes such as cell adhesion, cell migration, inflammation and cancer progression (Mochizuki and Okada, 2007; Reiss and Saftig, 2009; White, 2003). Much attention has been paid to the proteolytic activities of ADAMs, which can function as "molecular scissors" to shed membrane proteins from the cell surface. A large number of molecules have been identified as substrates for ADAMs, including extracellular matrix proteins, cell signaling receptors, growth factors and cytokines (Reiss and Saftig, 2009). A number of ADAMs are able to cleave the same substrates, for example, HB-EGF can be cleaved by ADAM9, ADAM12 and ADAM17, supporting the idea that ADAMs have many overlapping functions (Blobel, 2005). Loss-of-function studies in different species have provided further evidence for redundant biological functions of at least some ADAMs (Hoiruchi and Blobel, 2005; Jarriault and Greenwald, 2005). Therefore, obtaining a complete list of ADAM family members expressed in specific cells and tissues is critical to understanding the functions and physiological relevance of individual ADAM proteins. In this study we report the identification of a novel ADAM protein (ADAM41) with homology to ADAM10, which is a key sheddase involved in Notch signaling in both invertebrate and vertebrate species (Hartmann et al., 2002; Pan and Rubin, 1997).

\section{Results}

Identification of an ADAM41 metalloproteinase in X. tropicalis

We previously identified the $X$. tropicalis ortholog of mammalian ADAM10 (Wei et al., 2010). In the current study we used the $X$. tropicalis ADAM10 protein sequence to query the $X$. tropicalis genome and EST databases for potential paralogs of ADAM10 in this species. A partial genome sequence with relatively high similarity

Abbreviations used in this paper: ADAM, a disintegrin and Metalloproteinase); RTPCR (real time polymerase chain reaction); RACE (rapid amplification of cDNA ends); st. (stage).

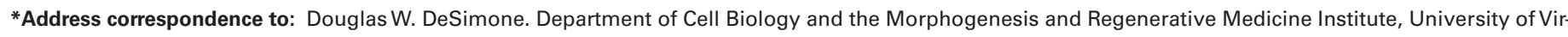

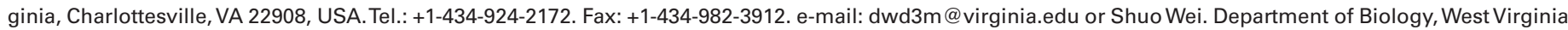
University, Morgantown, WV, USA.Tel.: +1-304-293-2106. e-mail: Shuo.Wei@mail.wvu.edu
} 
to ADAM10 was identified using this method. The sequence was used to design primers and the full-length cDNA sequence of a new gene was obtained subsequently by RT-PCR and RACE (GenBank Accession\# ADK56772). We named this new protein ADAM41 (also referred to as ADAM10-like; Wei et al., 2010). Although closest to ADAM10 among all paralogues identified to date in $X$. tropicalis,
ADAM41 is clearly encoded by a distinct gene based on overall nucleotide and predicted amino acid (42\%) sequence identities.

ADAM41 protein contains all of the typical domains of members of the ADAM family, including a signal sequence, a pro-domain, metalloproteinase, disintegrin, and cysteine-rich domains, a single transmembrane domain, and a cytoplasmic tail (Fig. 1 and Fig. 2C). Notably, ADAM41 has a conserved zinc-binding motif within its metalloproteinase domain that is required for ADAM metalloproteinase activity (Fig. 1), suggesting that the encoded protein is an active protease. All mammalian ADAMs, except for ADAMs 10 and 17, contain an EGF-like domain between the cysteine-rich and transmembrane domains (Janes et al., 2005). Our domain analyses suggest that, similar to ADAMs 10 and 17, X. tropicalis ADAM41 lacks an EGF-like sequence (not shown). This result is consistent with our hypothesis that ADAMs 10,17 and 41 belong to a subfamily of ADAMs that likely evolved from the same ancestor (Wei et al., 2010).

Phylogenetic analyses of the full length and the metalloproteinase domains of ADAM41, ADAM10 and ADAM17 from different species are shown in Fig. 2A-B. Putative orthologs of ADAM41 were found in zebrafish and non-placental mammals such as the duckbill platypus (Ornithorhynchus anatinus) and the opossum (Monodelphis domestica). However, these orthologs of ADAM41 are more divergent across species (40\% - 50\% amino acid identity/similarity) relative to either ADAM10 or ADAM17 (i.e., the two most closely related ADAM paralogs of ADAM41). Individual domains of $X$. tropicalis ADAM10 and ADAM41 were compared with those of human ADAM10 (Fig. 1 and Fig. 2C). The orthologous relationship between $X$. tropicalis and human ADAM10 is supported by a high degree of sequence similarity in each domain. In particular, the metalloproteinase domain and the cytoplasmic tail share more than $90 \%$ identity between human and $X$. tropicalis ADAM10, suggesting that the functions of these two domains are highly conserved.

Fig. 1. Amino acid comparisons among $X$. tropicalis ADAM41 (XtAD41), X. tropicalis ADAM10 (XtAD10), and human ADAM10 (HuAD10). Individual domains are indicated by horizontal arrow lines: signal peptide (SP), prodomain (PRO), metalloproteinase domain (MET), disintegrin domain (DIS), cysteine-rich domain $(C R)$, the transmembrane (TM) and the cytoplas mic tail (CYTO). The consensus HExGHxxGxxHD catalytic site motif is indicated by the asterisks. The open triangle indicates the invariant methionine in the Met-turn. 
By contrast, a comparison between $X$. tropicalis ADAM41 and human ADAM10 shows only $40-50 \%$ identity in all domains with even lower similarity ( $\sim 9 \%$ identical) for the cytoplasmic tail (Fig. $2 \mathrm{C})$. The amino acid identity of each domain between $X$. tropicalis ADAM41 and $X$. tropicalis ADAM10 is also in the range of $40-50 \%$ (Fig. $2 \mathrm{C}$ ), and there is no significant similarity between the sequences of their cytoplasmic tail domains. Thus, ADAM41 is a novel gene whose protein sequence shares the greatest, albeit relatively low, similarity to that of ADAM10 among all of the known ADAM sequences.

\section{Developmental expression of the $\mathrm{X}$. tropicalis adam41 gene}

The expression profile of $X$. tropicalis adam41 mRNA was assessed in embryos at different developmental stages by in situ hybridization. Maternal adam41 mRNA was detected at the animal pole of early stage embryos (Fig. 3A), whereas zygotic transcript was localized dorsally at gastrula stages (Fig. 3B) and concentrated along the neural plate at neurula stages (Fig. 3 C-D). As the embryos developed into later stages, adam41 mRNA was expressed diffusely in multiple tissues including brain, eye, branchial arches and somites (Fig. 3E-F).

\section{Knockdown of ADAM41 results in delayed neural development}

To further characterize the function of ADAM41 in $X$. tropicalis, an antisense morpholino oligodeoxynucleotide was designed to block the translation of adam41 mRNA(Fig. 4A). The efficacy of morpholino targeting was first established by determining the efficiency of translational inhibition following co-injection of antisense or control morpholinos in combination with transcripts encoding a myc-tagged ADAM41 protein. As indicated in Fig. 4B, ADAM41 antisense morpholino blocked translation of ADAM41, while the standard control morpholino had no effect. We were unable to determine the efficiency of the ADAM41 morpholino on endogenous ADAM41 protein, because a suitable ADAM41 antibody is not available.

It has been reported that vertebrate ADAM10 (and Kuzbanian, the ADAM10 homolog in Drosophila) plays an essential role during neurogenesis (Pan and

Fig. 2. $X$. tropicalis ADAM41 is a novel gene found in multiple vertebrate species, including non-placental mammals. (A,B) Phylogenetic analyses of full-length (A) and the metalloproteinase domains (B) of ADAMs 10, 17 and 41. Bootstrap values are indicated at major clades. Other clades with bootstrap values $>700$ are marked with stars. ADAM41 was identified in different species including zebrafish and X. tropicalis. However, non-placental mam-

mals, such as opossum and duck-billed platypus, were the only mammalian species identified to have ADAM41. ADAM10 and ADAM17 were found in all species analyzed. See experimental procedures for abbreviation of species. (C) X. tropicalis ADAM41 contains all of the signature domains of ADAM metalloproteinases. Each domain of X. tropicalis ADAM41 was compared to X. tropicalis and human ADAM10. The numbers listed above each domain indicate the percent identity compared to human ADAM10; the numbers in parentheses indicate the percent identity compared to X. tropicalis ADAM10.
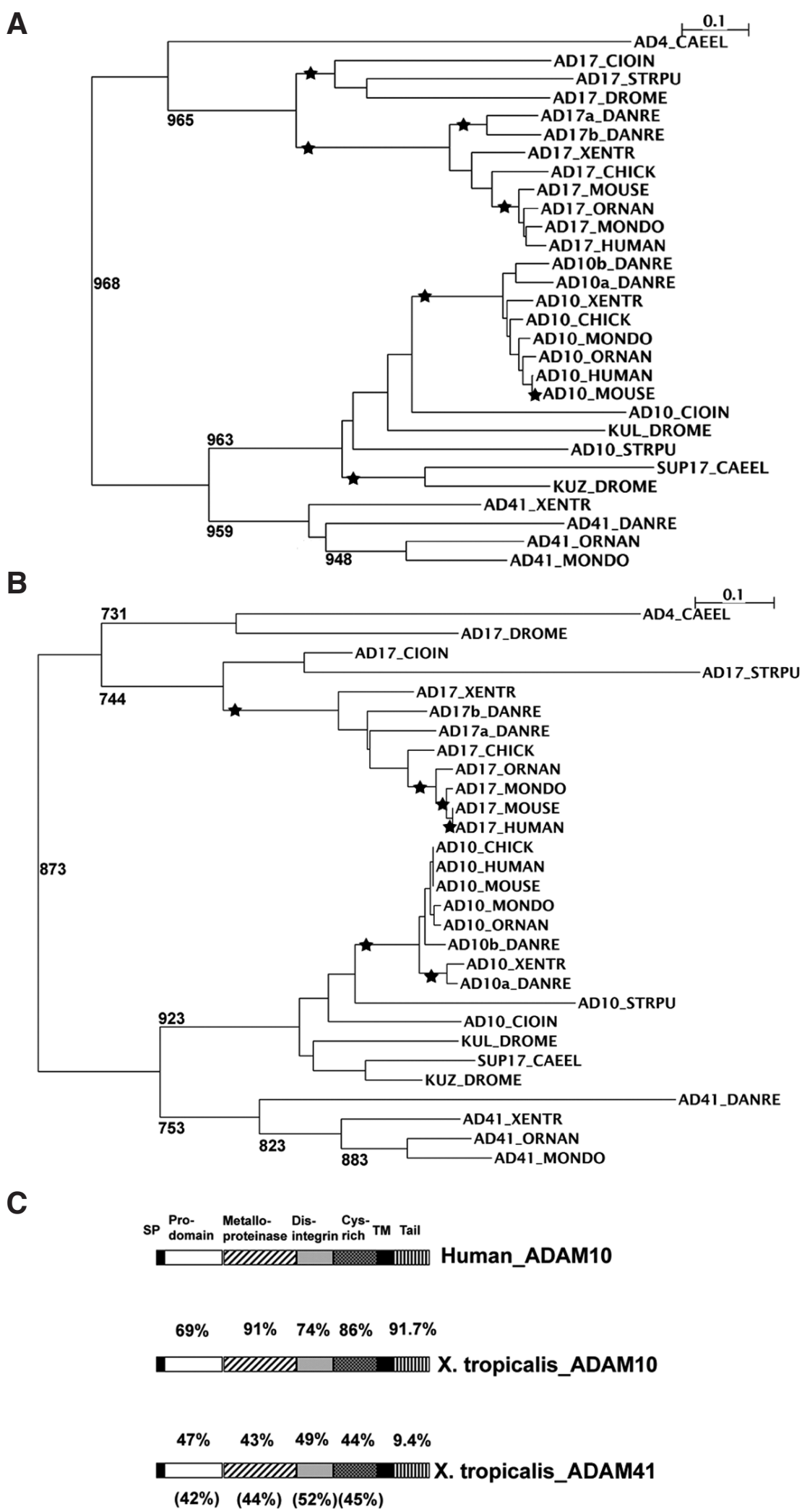

$(42 \%) \quad(44 \%)(52 \%)(45 \%)$ 


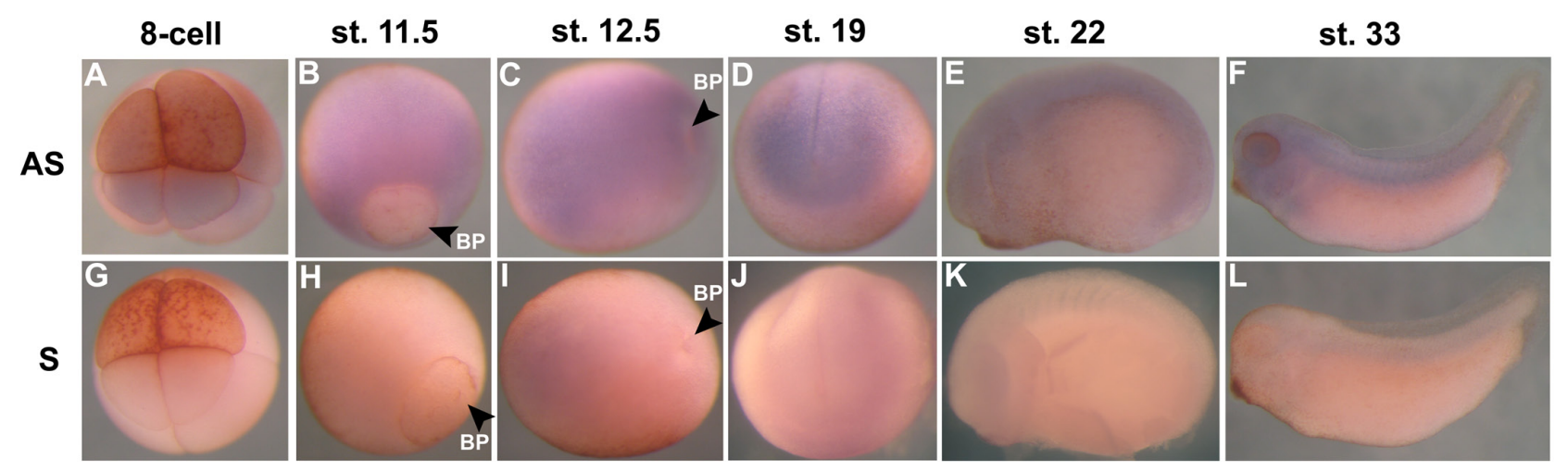

Fig. 3. Whole-mount in situ hybridization for adam41. Sense (S) and antisense (AS) transcripts of adam41 were used as probes for whole-mount in situ hybridization to detect the expressions of adam41 messenger at different developmental stages indicated. (A,G) Animal pole view; (B,C,H,I) dorsal view; (D,J) anterior-dorsal view; $(\mathbf{E}, \mathbf{F}, \mathbf{K}, \mathbf{L})$ side view. Arrowheads point to the blastopore (BP).

Rubin, 1997; Rooke et al., 1996). Therefore, we were interested in investigating whether ADAM41 is also involved in neural development in $X$. tropicalis. We used the proneural gene $n g n r-1$ and the neuron-specific gene $\mathrm{N}$-tubulin as markers to monitor the patterning of early neural development in X. tropicalis. Ngnr-1 is one of the earliest proneural genes that regulate neural fate determination in Xenopus (Ma et al., 1996)(Fig. 5A). Its expression is first detected at st. 11.5 and defines the prospective territory of primary neurons (Fig. 5F, arrows) and anterior facial neural placodes (Fig. 5G); as embryos develop, the anterior expression of $n g n r-1$ is restricted to neural placodes including the trigeminal ganglion (Fig. $5 \mathrm{H}, \mathrm{J}$, arrowhead). $\mathrm{N}$-tubulin is a neuronal differentiation marker whose expression spatially overlaps that of $n g n r-1$ but is temporally delayed (Chitnis et al., 1995; Ma et al., 1996). The earliest stage at which we detected the expression of $N$-tubulin was st.12.5 (Fig. $5 \mathrm{C}$ ), when $\mathrm{N}$-tubulin is expressed in loosely packed cells that are arranged in three parallel stripes demarcating the medial $(\mathrm{m})$, intermediate (i), and lateral neurons (I) on each side of the midline (Fig. 4 B-C). As the neural plate undergoes convergent extension and neural tube folding, these stripes shift and eventually locate at the ventral, intermediate and dorsal positions of the neural tube, where they differentiate into primary motor neurons, interneurons and sensory neurons, respectively (Fig. $5 \mathrm{C}-\mathrm{E}$ ). Transcripts of $\mathrm{N}$ tubulin are also expressed in the trigeminal ganglion at st.13 (Fig. 5B, arrowhead).

To test whether ADAM41 regulates neurogenesis, we injected ADAM41 morpholino into one blastomere of 2-cell stage embryos and examined patterns of neurogenesis by whole mount in situ hybridization using an ngnr-1 probe. Knockdown of ADAM41 caused a reproducible delay in neurogenesis relative to control morpholino and uninjected embryos. At st.12, for example, the expression of ngnr-1 was barely detected on the morpholino-injected side, whereas on the uninjected side ngnr-1 expression was normal, i.e., highly expressed in the prospective trigeminal ganglion (Fig. 6A, arrowhead). Ngnr-1 expression on the morpholino-injected side was delayed by approximately a half of a developmental stage relative to controls (Fig. 6C). This resulted in an asymmetry in ngnr-1 expression in the trigeminal ganglia at st.13; although the level of ngnr- 1 had already decreased on the uninjected side, it remained high on the side injected with the ADAM41 morpholino (Fig. 6E arrowheads). $N$-tubulin staining also confirmed the observed overall delay in neurogenesis (Fig. 6 L,M). At st.13, $N$-tubulin expression was barely detectable in the trigeminal ganglia on the morpholino injected side of the embryos, whereas on the uninjected side the staining was already apparent (Fig. 6L). Dorsal views of these embryos also revealed that the distance between the lateral stripe of neurons and the midline on the injected side was significantly increased (Fig. 6M, arrows), further indicating a general delay in neurogenesis (Fig. 6M). The observed delay in neurogenesis did not impair neural tube closure or the location of primary neurons in the neural tube (data not shown). The control morpholino used in these experiments did not cause neurogenesis delays in our experiments. Both injected and uninjected sides of these embryos retained normal spatiotemporal patterns of expression for both ngnr-1 and N-tubulin (Fig. 6 F-I).

We next asked whether the delay in neurogenesis was specific

A $\begin{array}{ll}\text { CTAGg CCAGCACAATGTTCTCATTT CAGCATTTC } & \text { ADAM41 } \\ \text { 5'TGCTGAAATGAGAACATTGTGCTGG }^{3} & \text { ADAM41 MO }\end{array}$

B

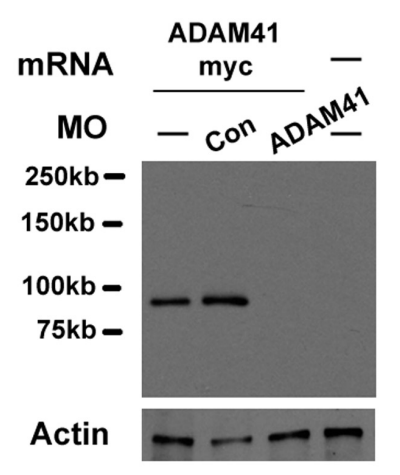

Fig. 4. Inhibition of ADAM41 translation by a specific antisense morpholino. (A) A morpholino (MO) was designed to target underlined sequence covering the translational start site (indicated in red) in adam41 mRNA. (B) ADAM41 morpholinos blocked the translation of myc-tagged exogenous ADAM41 mRNA. The indicated morpholinos were co-injected with exogenous myc-tagged adam41 mRNA into X. tropicalis embryos at one-cell stage, and Western blot was carried out using an anti-myc antibody. Each lane contains 10 embryo equivalent of total protein. 
A

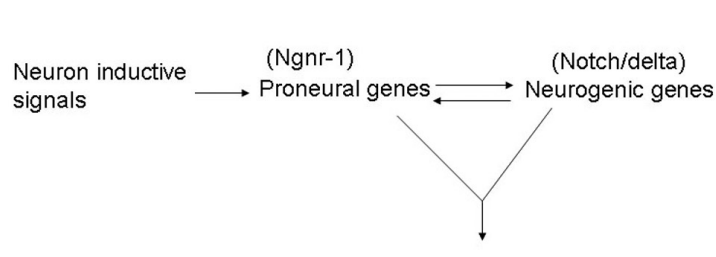

Differentiated neurons

(N-tubulin)
B st.13 N-tubulin

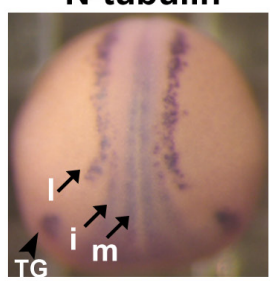

Fig. 5. In situ hybridization of neural markers in normal $X$. tropicalis embryos. (A) Neural induction in Xenopus involves multiple events. Early neural induction signals activate proneural genes that specify neural fate of ectodermal cells. Ngnr-1 is a strongly expressed proneural gene in X. tropicalis. Proneural genes coordinate with neurogenic genes, such as notch/delta, to define neuronal pattern. (B-E) Dorsal view of X. tropicalis embryos demonstrating primary neuron patterning as seen by whole-mount in situ hybridization for the neuron marker $\mathrm{N}$-tubulin. Three stripes of neurons, medial neurons (m), intermediate neurons (i), and lateral neurons (I), form in parallel to each other (arrows in B and C) on both sides of dorsal midline axis. Arrowhead in (B) points to the trigeminal ganglion (TG) in the anterior neural plate of the embryo. (F-J) Whole-mount in situ hybridization for the proneural gene ngnr-1 in X. tropicalis embryos at different stages. (F) Ngnr-1 message is first expressed dorsally as three stripes on each side of the midline at st. 11.5 (arrows denote dorsal ngnr-1 expression, and star indicates blastopore surrounding circular yolk plug). At this stage ngnr-1 is also expressed in the anterior region (G, anterior view of embryo with dorsal side at the top). The dorsal stripes elongated and migrated toward the midline st.13 as the embryos developed (1, arrow). Anterior ngnr-1

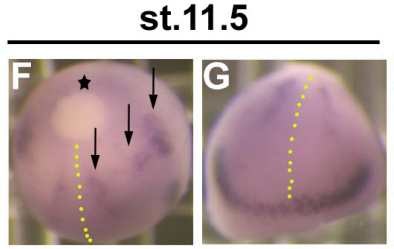

st.12
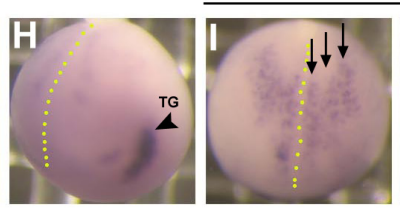
expression decreased and was restricted to neural placodes including the trigeminal ganglion (TG) in st.12/13 embryos (arrowheads in $\mathrm{H}$ and $\mathrm{J}$; embryo in $\mathrm{H}$ is tilted to the left in order to give a better view of anterior right side TG staining). The yellow dashed line defines the midline of each embryo.

to ADAM41 or was a nonspecific consequence of the morpholino injection. To test this, we prepared an ADAM41 "rescue" transcript by altering the specific target sequence recognized by the ADAM41 morpholino. Co-injection of the ADAM41 rescue transcript with the ADAM41 antisense morpholino into one blastomere of 2-cell stage embryos rescued the delay (Fig. 7 A-D). Spatial and temporal expression of both the presumptive trigeminal ganglia (Fig. 7 B,D arrowheads) and lateral neuronal stripes could be restored by expression of exogenous ADAM41. The efficiency of the rescue was dependent on the amount of the ADAM41 rescue transcript co-injected (Fig. 7E). These data suggest that ADAM41 is required for the normal temporal progression of neurogenesis.

\section{Ngnr-1}

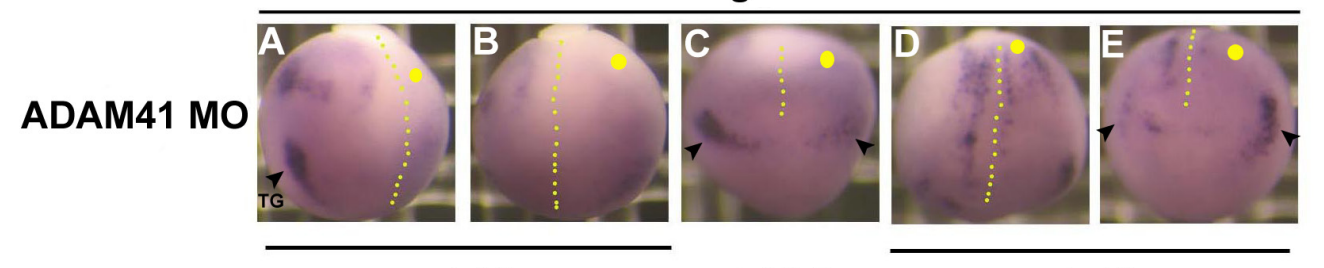

st.12

st.12.5

N-tubulin, st. 13

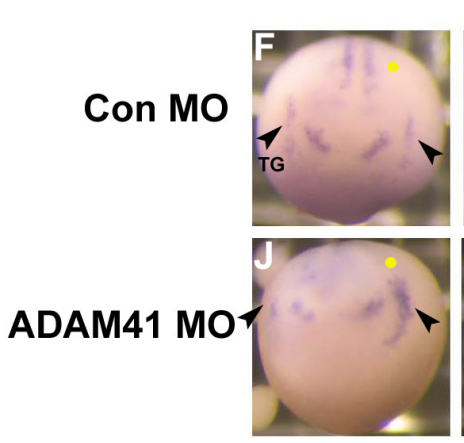

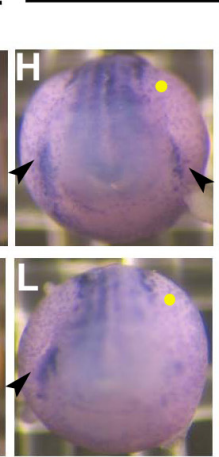

Fig. 6. Knockdown of ADAM41 caused a developmental delay at neurula stages. Embryos were injected in one cell at 2-cell stage with the indicated morpholinos. Injected side is marked by yellow dot in each panel, and dotted yellow lines indicate midline position in (A-E). (A-E) Injection of ADAM41 morpholino resulted in a delay of ngnr-1 expression pattern in the trigeminal ganglia at late gastrula stages. The side injected with ADAM41 morpholino had reduced ngnr-1 expression while the uninjected side had normal ngnr-1 expression pattern at st. 12 ( $A, B$ are different views of the same embryo). The ADAM41 morpholino injected side started to express ngnr-1 at st.12.5 (C) (anterior view). By stage 13, expression of ngnr-1 in the trigeminal ganglion at the uninjected side had significantly decreased ( $D, E$ are different views of the same embryo). In contrast, ngnr-1 was more pronounced in the ADAM41 morpholino injected side compared with uninjected side at st. 13; see arrowheads in (E). (F-M) Effects of ADAM41 knockdown on the expression of ngnr-1 and $\mathrm{N}$-tubulin at st. 13. Control morpholino did not alter the early patterns of primary neurons $(\mathbf{G}, \mathbf{I})$ or anterior neural placodes (F,H). ADAM41 knockdown resulted in an enlarged expression domain of ngnr-1 (J) and decreased N-tubulin staining in trigeminal ganglion (L), typical of earlier stage control or uninjected embryos[SW1]. The distance between the lateral neuronal stripe and midline increased compared to the uninjected side (compare arrows in $M$ with those in I). 

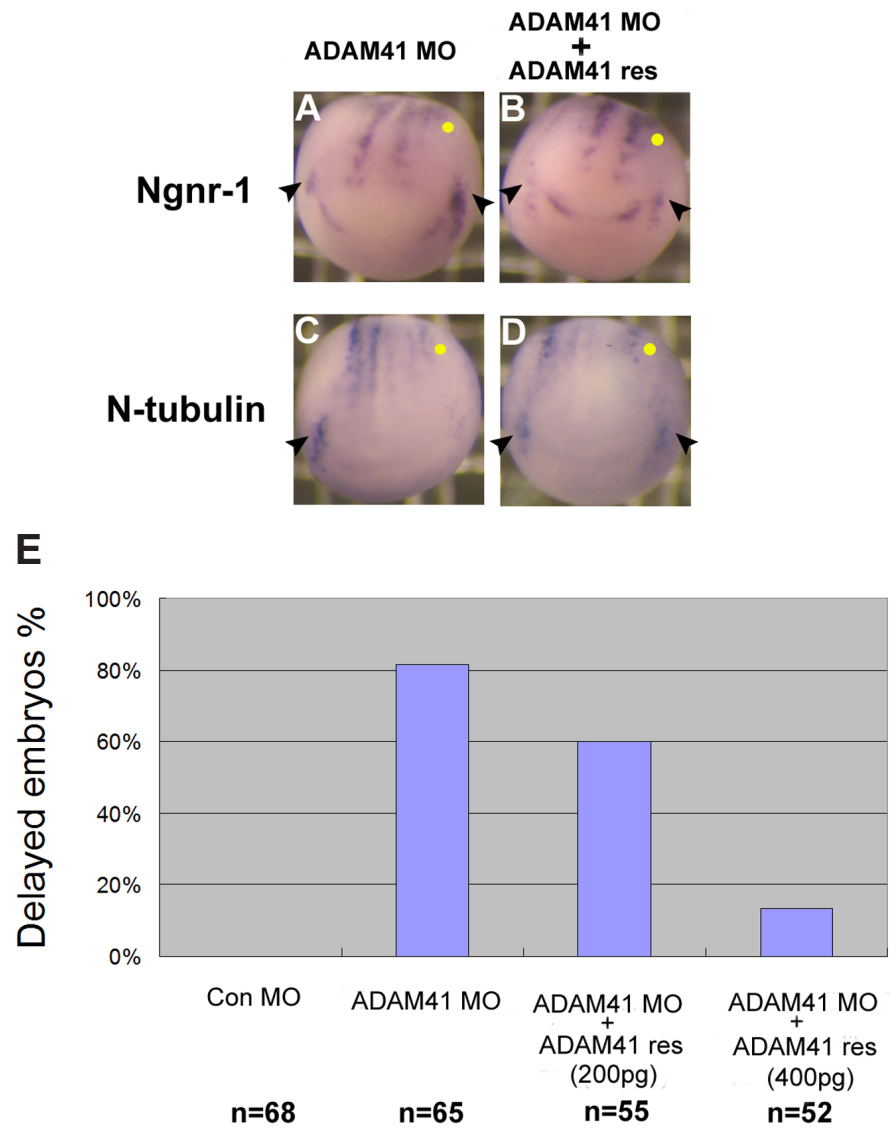

Fig. 7. Developmental delay phenotype caused by knockdown of ADAM41 was rescued by an exogenous ADAM41 transcript. (A-D) Co-injection of the ADAM41 rescue transcript into one cell of 2-cell stage embryos restored ngnr-1 and $\mathrm{N}$-tubulin expression in trigeminal ganglia in ADAM41 morphants (B,D). Arrowheads point to the trigeminal ganglia, and the injected side is denoted with a yellow dot in each panel. (E) Summary of the effect of ADAM41 rescue transcript on the expanded ngnr-1 expression caused by ADAM41 morpholino. More than $80 \%$ of ADAM41 morphants displayed expandedngnr-1 expression in the trigeminal ganglion. Co-injection of 200pg of ADAM41 rescue transcript reduced the number of abnormal embryos to $60 \%$, and less than $20 \%$ embryos showed expanded ngnr-1 expression in the trigeminal ganglion when 400pg rescue transcript was used.

\section{Discussion}

In this study, we identified a novel ADAM protease, ADAM41, bearing all signature ADAM domains. ADAM41 also contains the consensus HExGHxxGxxHD catalytic site motif, suggesting that the protein is proteolytically active. Putative homologies of ADAM41 are found in several vertebrates, including teleosts and non-placental mammals. Interestingly, we did not detect ADAM41 in placental mammals or in birds. Among the invertebrates, Drosophila has both the adam10 ortholog (Kuzbanian) and a close paralogue Kul (Kuzbanian-like) gene (Sapir et al., 2005). In our phylogenetic analyses (Fig. $2 \mathrm{~A}-\mathrm{B}$ ), Drosophila $\mathrm{Kul}$ is in the ADAM10 clade whereas ADAM 41 forms a different clade, suggesting that ADAM41 is a novel ADAM distinct from ADAM10 and Kul. We were unable to find evidence for ADAM41 in nematodes, the tunicate Ciona or other invertebrates for which sequence information is available. By contrast, ADAM10 and ADAM17 are highly conserved in nematodes, Ciona, Drosophila and the vertebrates. The variable distribution of ADAM41 orthologs in the Metazoa suggests possible dedicated functions for ADAM41 in those animals (e.g., amphibians, teleosts and non-placental mammals) in which it is expressed.

ADAM10 has been reported to regulate neurogenesis through proteolytic processing of the Notch receptor and/or its ligand delta in Drosophlia (Pan and Rubin, 1997; Rooke et al., 1996). Data obtained by overexpression of a dominant negative form of mouse ADAM10 in Xenopus resulted in apparent hyperplasia of neurons, suggesting that ADAM10 might function the same way in frog as it does in Drosophlia to regulate neurogenesis (Pan and Rubin, 1997). Although the ADAM41 protein sequence is most similar to ADAM10, we did not observe any obvious Notch-related phenotype (e.g., neuronal hyperplasia) in $X$. tropicalis embryos following knockdown of ADAM41. However, ADAM41 morphants did display a specific delay in neurogenesis, which could be rescued by co-injecting a non-targetable ADAM 41 transcript. This suggests that ADAM41 is required, either directly or indirectly, for the timing of neurogenesis in $X$. tropicalis. In some scenarios involving morpholino microinjections, general delays in developmental progression are observed at gastrulation, which can affect the timing of later events in neurulation and beyond. In our studies, embryos injected with ADAM41 morpholino went through gastrulation normally, suggesting that the observed delay in neurogenesis was not a consequence of a general delay in gastrulation. It has been reported that overexpression of cyclin and cyclin-dependent kinase in Xenopus embryos altered cell proliferation and resulted in delays in neurogenesis (Richard-Parpaillon et al., 2004). We did not observe abnormalities in cell proliferation in embryos injected with ADAM41 morpholino (data not shown), further suggesting that morpholino knockdown of ADAM41 delayed neurogenesis by a distinct mechanism not involving disruption of cell cycle. More studies will be needed to elucidate the mechanism behind the morphant phenotype.

\section{Materials and Methods}

\section{Phylogenetic analysis}

Sequence alignment and bootstrap trees (exclude positions with gaps; correct for multiple substitutions) were generated using ClustalX, and phylogenetic trees were drawn using Njplot. Domain structures of ADAM41, ADAM10 and ADAM17 were predicted by the Simple Modular Architecture Research Tool (http://smart.embl-heidelberg.de/).

The following ADAM protein sequences were used to generated the phylogenetic trees: $X$. tropicalis (XENTR) ADAM10 (ABB92398), $X$ tropicalis ADAM41 (ADK56772), $X$. tropicalis ADAM17 (ADK56773), Drosophila(DROME) Kuz (AAC47275), DrosophilaKul (AAF56926), Drosophila ADAM17 (AAO53296), zebrafish (DANRE) ADAM10a (NP_001152786), zebrafishADAM10b (NP_956714), zebrafishADAM41 (XP_689465), zebrafish ADAM17a (AAH57410), zebrafish ADAM17b (XP_689147), C.elegans (CAEEL) Sup-17/ADAM10 (AAB97161), C.elegans ADM-4/ADAM17 (AAB09097), Ciona Intestinalis (CIOIN) ADAM17 (XP_002122475), Ciona Intestinalis ADAM10 (XP_002127224), sea urchin (STRPU) ADAM17 (XP_001185879), sea urchin ADAM10 (XP_001177064), human ADAM10 (AAC51766), human ADAM17 (AAI36784), mouse ADAM10 (CAX15709), mouse ADAM17 (AAI38417), chicken ADAM10 (AAL77215), chicken ADAM17 (AAS64567), opossum (MONDO) ADAM10 (XP_001377651), opossum ADAM41 (XP_001376025), opossum ADAM17 (XP_001381171), duck-billed platypus (ORNAN) ADAM10 (XP_001506337), duck-billed 
platypus ADAM41 (XP_001507234), duck-billed platypus ADAM17 (XP_002758088).

\section{Cloning of the X. tropicalis ADAM41 gene}

Primers 5'-TGAAATACTATGAAAACCTC-3' (forward) and 5'- AATAAACACAGTGCCAGC-3' (reverse) were used to amplify by PCR a partial sequence of adam 41 from a cDNA library made from $X$. tropicalis embryos at st. 13. The full-length sequence of adam 41 was subsequently obtained by 5'RACE and 3'RACE using the SMART RACE kit (Clontech). Primers 5'-ACACAGTGCCAGCCATTAACGCAGCC-3' and 5'-ACACTGGCTCATGAATTAGGCCACAGCC-3' were used for 5'-RACE and 3'-RACE, respectively.

All PCR products of adam41 were sequenced by the Biomolecular Research Facility at the University of Virginia.

\section{Embryo manipulation, injection and Western blot}

Wild-type $X$. tropicalis adults were purchased from NASCO. Embryos were obtained by natural mating following protocols from Dr. Robert Grainger's lab (http://faculty.virginia.edu/xtropicalis/) and staged according to Nieuwkoop and Faber (1967). Morpholino oligodeoxynucleotides were designed and synthesized by Gene Tools and injected as described (Ogino et al., 2006). Red dextran was co-injected as a tracer. Anti-Myc antibody (9E10) was obtained from Santa Cruz Biotechnology. ADAM41 rescue transcript was produced by PCR using a forward primer 5'-ccatgttTAGTttCcaAcatttcaattttatatggttg-3' (nucleotide substitutions indicated in caps, and translation initiation underlined) designed to mutate the antisense morpholino target sequence but still encode a start site and portion of the protein.

\section{Whole-mount in situ hybridization}

Digoxygenin-labeled antisense probes for adam10, adam41 (full-length), $n g n r-1$ and $\mathrm{N}$-tubulin were prepared by in vitro transcription. Embryos were fixed, hybridized and stained as described (Sive et al., 2000).

\section{Acknowledgements}

We thank Fred Simon, Tania Rozario and Maureen Bjerke for assistance with X. tropicalis husbandry, and the rest of the DeSimone lab for inspiring discussions. We are also grateful to Drs. Tyra Wolfsberg and Charles Whittaker for advice on sequence and phylogenetic analyses. This work was supported by the NIH (DE14365/GM094793 to D.W.D) and the March of Dimes Foundation (F405-140 to J.M.W. and 1-FY10-399 to D.W.D and S.W.).

\section{References}

BLOBEL, C.P. (2005). ADAMs: key components in EGFR signalling and development. Nat Rev Mol Cell Biol 6: 32-43.

CHITNIS, A., HENRIQUE, D., LEWIS, J., ISH-HOROWICZ, D. and KINTNER, C. (1995). Primary neurogenesis in Xenopus embryos regulated by a homologue of the Drosophila neurogenic gene Delta. Nature 375: 761-766.
EDWARDS, D.R., HANDSLEY, M.M. and PENNINGTON, C.J. (2008). The ADAM metalloproteinases. Mol Aspects Med 29: 258-289.

HARTMANN, D., DE STROOPER, B., SERNEELS, L., CRAESSAERTS, K., HERREMAN, A., ANNAERT, W., UMANS, L., LUBKE, T., LENA ILLERT, A., VON FIGURA, K. et al., (2002). The disintegrin/metalloprotease ADAM 10 is essential for Notch signalling but not for alpha-secretase activity in fibroblasts. Hum $\mathrm{Mol}$ Genet 11: 2615-2624.

HOIRUCHI, K. and BLOBEL, C.P. (2005). Studies From ADAM Knockout Mice. In The ADAM Family of Proteases, (ed. HOOPER, N. M. and LENDECKEL, U.). Springer, pp.29-64.

JANES, P.W., SAHA, N., BARTON, W.A., KOLEV, M.V., WIMMER-KLEIKAMP, S.H., NIEVERGALL, E., BLOBEL, C.P., HIMANEN, J.P., LACKMANN, M. and NIKOLOV, D.B. (2005). Adam meets Eph: an ADAM substrate recognition module acts as a molecular switch for ephrin cleavage in trans. Cell 123: 291-304.

JARRIAULT, S. and GREENWALD, I. (2005). Evidence for functional redundancy between C. elegans ADAM proteins SUP-17/Kuzbanian and ADM-4/TACE. Dev Biol 287: 1-10.

MA, Q., KINTNER, C. and ANDERSON, D.J. (1996). Identification of neurogenin, a vertebrate neuronal determination gene. Cell 87: 43-52.

MOCHIZUKI, S. and OKADA, Y. (2007). ADAMs in cancer cell proliferation and progression. Cancer Sci 98: 621-628.

OGINO, H., MCCONNELL, W.B. and GRAINGER, R.M. (2006). High-throughput transgenesis in Xenopus using I-Scel meganuclease. Nat Protoc 1: 1703-1710.

PAN, D. and RUBIN, G.M. (1997). Kuzbanian controls proteolytic processing of Notch and mediates lateral inhibition during Drosophila and vertebrate neurogenesis. Cell 90: 271-280.

REISS, K. and SAFTIG, P. (2009). The "a disintegrin and metalloprotease" (ADAM) family of sheddases: physiological and cellular functions. Semin Cell Dev Biol 20: 126-137.

RICHARD-PARPAILLON, L., COSGROVE, R.A., DEVINE, C., VERNON, A.E. and PHILPOTT, A. (2004). G1/S phase cyclin-dependent kinase overexpression perturbs early development and delays tissue-specific differentiation in Xenopus. Development 131: 2577-2586.

ROOKE, J., PAN, D., XU, T. and RUBIN, G.M. (1996). KUZ, a conserved metalloprotease-disintegrin protein with two roles in Drosophila neurogenesis. Science 273: $1227-1231$

SAPIR, A., ASSA-KUNIK, E., TSRUYA, R., SCHEJTER, E. and SHILO, B.Z. (2005). Unidirectional Notch signaling depends on continuous cleavage of Delta. Development 132: 123-132.

SIVE, H.L., GRAINGER, R.M. and HARLAND, R.M. (2000). Early Development of Xenopus laevis: A Laboratory Manual. Cold Spring Harbor Laboratory Press.

WEI, S., WHITTAKER, C.A., XU, G., BRIDGES, L.C., SHAH, A., WHITE, J.M. and DESIMONE, D.W. (2010). Conservation and divergence of ADAM family proteins in the Xenopus genome. BMC Evol Biol 10: 211.

WHITE, J.M. (2003). ADAMs: modulators of cell-cell and cell-matrix interactions. Curr Opin Cell Biol 15: 598-606.

WHITE, J.M., BRIDGE, L.C., DESIMONE, D.W., TOMCZUK, M. and WOLFSBERG, T. (2005). Introduction to the ADAM Family. In The ADAM Family of Proteases, (ed. HOOPER, N. M. and LENDECKEL, U.). Springer, pp.1-28. 


\section{Further Related Reading, published previously in the Int. J. Dev. Biol.}

ADAM17 overexpression promotes angiogenesis by increasing blood vessel sprouting and pericyte number during brain microvessel development

Juntang Lin, Cornelius Lemke, Christoph Redies, Xin Yan, Eilhard Mix, Arndt Rolfs and Jiankai Luo Int. J. Dev. Biol. (2011) 55: 961-968

Matrix metalloproteinase-2 is involved in the migration and network formation of enteric neural crest-derived cells

Richard B. Anderson

Int. J. Dev. Biol. (2010) 54: 63-69

Neural stem cells at the crossroads: MMPs may tell the way

Gaetana A. Tonti, Ferdinando Mannello, Emanuele Cacci and Stefano Biagioni Int. J. Dev. Biol. (2009) 53: 1-17

Soluble membrane-type 3 matrix metalloprioteinase causes changes in gene expression and increased gelatinase activity during Xenopus laevis development Logan A. Walsh, Colin A. Cooper and Sashko Damjanovski

Int. J. Dev. Biol. (2007) 51: 389-396

Matrix metalloproteinases in cancer: from new functions to improved inhibition strategies Alicia R. Folgueras, Alberto M. Pendás, Luis M. Sánchez and Carlos López-Otín Int. J. Dev. Biol. (2004) 48: 411-424

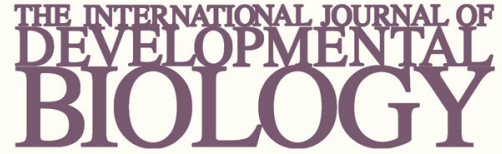

Volume 54 Nos. 6/7

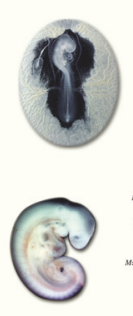

Developmental Hematopoiesis
Special Issue
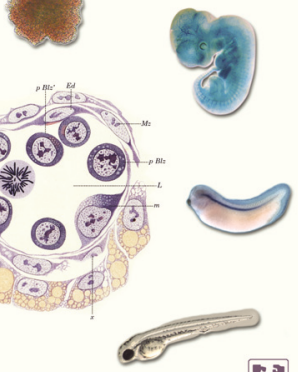

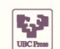

5 yr ISI Impact Factor $(2010)=2.961$

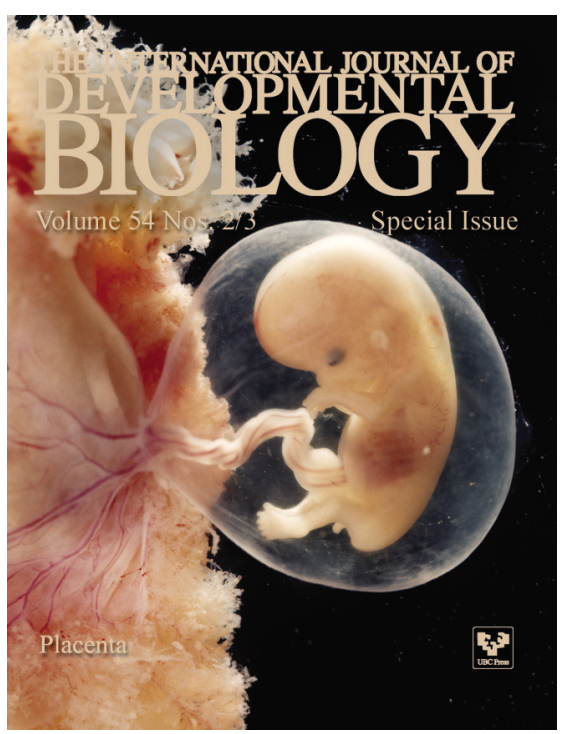

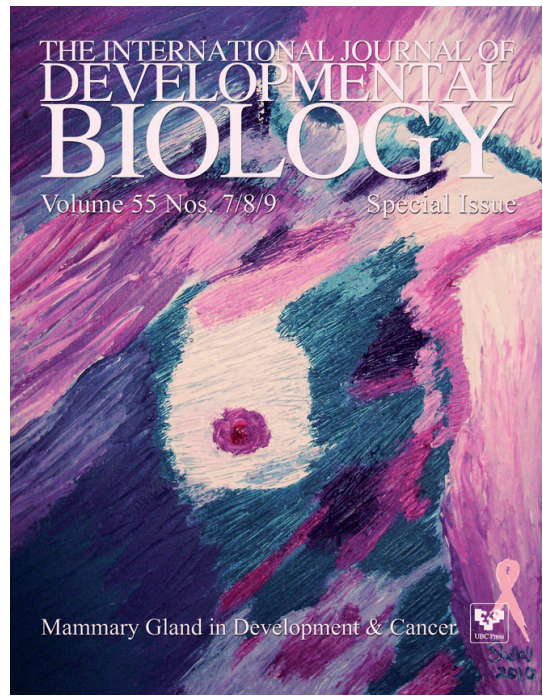

THE INTERNATIONAL IOURNAI OF
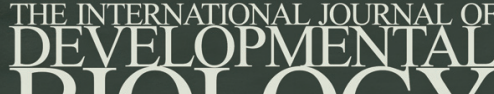
$\mathrm{BIO}$

Volume 55 Nos. $4 / 5$

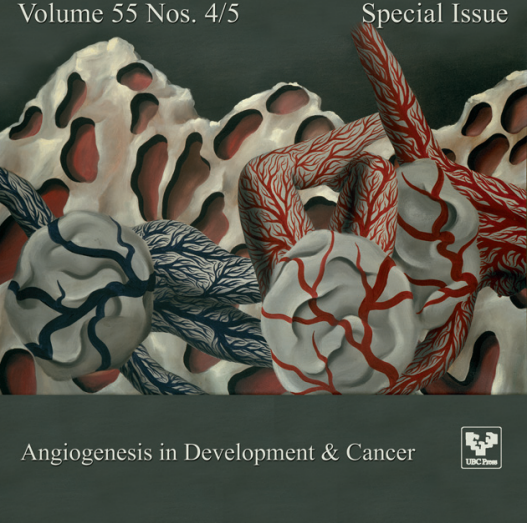

